cohort studies, and strong evidence to design robust policies to protect pregnant women from heat stress risks in developing countries for improved reproductive health.

\section{0-371 OCCUPATIONAL HEAT EXPOSURES AND RENAL HEALTH IMPLICATIONS - A CROSS-SECTIONAL STUDY AMONG COMMERCIAL KITCHEN WORKERS IN SOUTH INDIA}

'Vidhya Venugopal, Latha PK, Rekha Shanmugam. 'Sri Ramachandra Institute of Higher Education and Research, India

\subsection{6/OEM-2021-EPI.57}

Introduction Strenuous jobs in hot working environments, such as in commercial kitchens, are proven risk factors for adverse renal implications for workers working without adequate interventions. Evidence is scarce on the heat exposures and their impacts on commercial kitchen workers' renal health.

Objectives To investigate the renal health implications due to the workers' exposures to heat stress and exertion in commercial kitchens.

Methods We conducted a cross-sectional study among 266 workers in 7-commercial kitchens in the summer and winter of 2018. We monitored the Wet Bulb Globe Temperatures (WBGT), the physiological indicators of heat strain viz., rise in Core Body Temperature (CBT), Sweat Rate (SwR), and Urine specific gravity (USG) and analyzed the post-shift serum creatinine to calculate the estimated Glomerular Filtration Rate (eGFR). We administered a validated questionnaire to capture the workers' self-reported renal health symptoms of heat stress.

Results About $66 \%$ of workers were exposed to WBGTs levels higher than the safer Threshold Limit Value (TLV) with an average exposure of $30.1^{\circ} \mathrm{C} \pm 2.7^{\circ} \mathrm{C}$. Among the exposed workers, $82 \%$ reported experiencing heat strain symptoms such as excessive sweating, exhaustion, headache irrespective of the season. Above TLV-WBGT exposures were significantly associated with self-reported symptoms of dehydration (Adjusted Odds Ratio (AOR):2.3; 95\% CI:1.2-4.3) and measured heat strain indicators (AOR: 2.9; 95\% CI:1.6-5.1). Prevalence of heat strain indicators viz., rise in CBT (9.1\%), SwR (17\%), USG (75\%), was observed among heat-exposed workers. Heat-exposed workers had a 2.8-fold higher risk of reduced kidney function (eGFR of $<90 \mathrm{~mL} / \mathrm{min} / 1.73 \mathrm{~m} 2$ ) even after adjusting for the potential confounders (AOR:2.8; 95\% CI:1.1-6.9).

Conclusion The preliminary study results show adverse renal impacts of heat exposures among commercial kitchen workers that warrant further investigation to arrive at conclusive results. A need for adaptation and interventions is imperative to protect few million kitchen workers from hazards of occupational heat stress.

\section{0-418 OCCUPATIONAL HEAT EXPOSURES, PHYSIOLOGICAL RESPONSES AND RENAL HEALTH OUTCOMES AMONG BRICK WORKERS IN SOUTH INDIA}

${ }^{1}$ PK Latha, Vidhya Venugopal, Rekha Shanmugam. ${ }^{1}$ AMET University, India
Introduction Rising temperature with consequent heat stress is likely to subject millions of workers exerting outdoors at risk of heat-related illnesses and adverse renal health outcomes. Need for such research evidence is urgently needed to address this issue especially in the changing climate scenario.

Objective To investigate the relationship between occupational heat stress, physiological indicators of heat strain, and associated renal health outcomes among brick workers in South India.

Methods We conducted a cross-sectional survey with 327 brick workers during the summer of $2017 \& 2018$. We collected Wet Bulb Globe Temperatures $\left(\mathrm{WBGT}^{\circ} \mathrm{C}\right.$ ), pre-and post-shift Core Body Temperature(CBT), Urine Specific Gravity(USG), and post-shift Sweat rate(SwR), and serum creatinine/uric acid for calculating eGFR (estimated Glomerular Filtration Rate), a kidney function indicator.

Results Workers were exposed to an average WBGT of $27.7^{\circ} \mathrm{C}$ \pm 2.2 with more than $51 \%$ of measurements above the ACGIH-Threshold Limit Value (TLV). 69\% of the workers reported heat-strain symptoms such as excessive sweating, exhaustion, and headaches. The workers exposed to above TLV-WBGT had 1.8-fold higher risk of dehydration (Adjusted Odds Ratio (AOR):1.8; 95\%CI: $1.0-3.0, \mathrm{p}=0.03$ ), rise in CBT $(\mathrm{AOR}=2.2, \mathrm{CI}: 1.0-4.7, \mathrm{p}=0.02)$ and measured heatrelated symptoms $(\mathrm{AOR}=2.5,95 \% \mathrm{CI}: 1.5-4.2, \mathrm{p}=0.0001)$. Heat-exposed workers had $\mathrm{CBT}>1{ }^{\circ} \mathrm{C} \quad(14 \%), \quad S w R>1$ lit/hr (24\%), and USG $>1.020$ (35\%). The prevalence of low eGFR ( ; 95\%CI: 1.6-4.2) compared to workers exposed to WBGT below TLV.

Conclusion The preliminary study results only give a clue to the impacts of occupational heat stress on renal health. To have conclusive results, further epidemiological investigations are warranted with stratification for various personal and exposure factors that determine the disease etiology. With or without evidence, the drive for precautionary protective labor policies/welfare measures does not diminish for better occupational health outcomes.

\section{0-427 RISK FACTORS FOR ELEVATED CORE BODY TEMPERATURE IN FARMWORKERS}

Chelsea Langer, 'Marc Schenker. ' Univ. of California at Davis, United States

\subsection{6/OEM-2021-EPI.59}

Introduction Heat-related illness (HRI) poses a serious occupational health risk for farmworkers, particularly considering harvest season corresponds to peak summertime heat. The California Heat Illness Prevention Study (CHIPS) collected objective data on the physiological responses of farmworkers to environmental heat, covering a wide range of crops and working conditions.

Objectives The primary objective of this analysis is to identify risk factors for elevated core body temperature (CBT).

Methods A convenience sample of farmworkers was recruited through farms and farm labor contractors. Bilingual researchers administered questionnaires pre- and post-shift. CBT and work rate were measured using an ingestible wireless thermistor and an accelerometer, respectively. Ambient weather conditions, including wet bulb globe temperature (WBGT), were recorded using two weather stations at the work site. Multiple 